\title{
THE HILBERT BALL AND BI-BALL ARE HOLOMORPHICALLY INEQUIVALENT ${ }^{1}$
}

\author{
BY STEPHEN J. GREENFIELD AND NOLAN R. WALLACH
}

Communicated by Irving Glicksberg, August 31, 1970

1. Introduction. In this note we prove that if $B$ is the unit ball of a complex Hilbert space then $B$ and $B \times B$ are holomorphically inequivalent. This answers a question of Burghelea. We also announce some results on the automorphism groups of bounded domains in a Hilbert space.

2. The ball and the bi-ball. Let $H$ be a complex Hilbert space. Let $B=\{z \in H \mid\|z\|<1\}$. Here $\|\cdots\|$ is the Hilbert norm, and we denote by $\langle$,$\rangle the inner product on H$.

THEOREM 2.1. $B$ and $B \times B$ are holomorphically inequivalent. (That is, there is no diffeomorphism $f: B \rightarrow B \times B$ so that $d f(z)$ is complex linear for each $z \in B$.)

Proof. Suppose that $f: B \rightarrow B \times B$ is a holomorphic equivalence. We derive a contradiction. We first assert that we may assume that $f(0)=(0,0)$. Indeed, suppose that $f(z)=(0,0), z \in B$. Define for $w \in B, w=w_{1}+\lambda z,\left\langle w_{1}, z\right\rangle=0$,

$$
h(w)=\frac{\left(1-\|z\|^{2}\right)^{1 / 2} w_{1}+(\lambda+1) z}{\lambda\|z\|^{2}+1} .
$$

It is not hard to check that $h: B \rightarrow B$ is a holomorphic self-equivalence and $h(0)=z$. Replace $f$ by $f \circ h$. Then $f(0)=(0,0)$.

Let $z \in B$. Then

$$
f(\lambda z)=\sum_{k=1}^{\infty}\left(\lambda^{k} / k !\right) d^{k} f(0) z^{k} \text { for }|\lambda| \leqq 1
$$

and the convergence is uniform on $|\lambda| \leqq 1$. (Here $d^{k} f(0)$ is the $k$ th derivative of $f, z^{k}$ is the $k$-tuple $(z, \cdots, z)$.)

Set

$$
G(z)=\frac{1}{2 \pi} \int_{0}^{2 \pi} e^{-i \theta f} f\left(e^{i \theta} z\right) d \theta .
$$

AMS 1969 subject classifications. Primary 5755, 3260, 2270.

Key words and phrases. Hilbert space, Banach space, unit balls, inequivalence of polyballs, Cartan domains, Siegel domains, Schwarz lemma.

1 Partially supported by the NSF through grants GP-20631 and GP-20647. 
Since $G(z)$ is a limit of convex combinations of elements of $B \times B$ bounded away from the boundary of $B \times B, G(z) \in B \times B$ for $z \in B$. Thus, by (1), $d f(0) B C B \times B$. Similarly, setting $T=d f(0)$, we have $T^{-1}(B \times B) \subset B$. Hence $T$ is a continuous linear bijection of $H$ with $H \times H$ so that $T: B \rightarrow B \times B$ is a bijection. Let $z \in H$ be a unit vector. Then $(z, 0)$ is in the boundary of $B \times B$. Clearly $T^{-1}(z, 0)=v$ is in the boundary of $B$. Hence $\|v\|=1$. Let $w \in B$ then $(z, 0)=\frac{1}{2}(z, w)$ $+\frac{1}{2}(z,-w),(z, w),(z,-w) \in(B \times B)^{-}$. Hence if $v_{1}=T^{-1}(z, w)$, $v_{2}=T^{-1}(z,-w)$ then $v_{1}, v_{2} \in \bar{B}$ and $v=\frac{1}{2} v_{1}+\frac{1}{2} v_{2}$. This is the desired contradiction. Q.E.D.

3. Automorphism groups of bounded domains in Hilbert space. In this section we announce several of the results of [1]. Let $H$ be a complex Hilbert space. Let $L\left(\boldsymbol{C}^{n}, H\right)$ be the space of all complex linear maps from $\boldsymbol{C}^{n}$ to $H$. Then $L\left(\boldsymbol{C}^{n}, H\right)$ is a Hilbert space relative to the inner product $(Z \mid W)=\operatorname{tr} W^{*} Z$. (Here we give $\boldsymbol{C}^{n}$ the standard Hilbert space structure; adjoints are denoted by an upper asterisk.) Let $D_{n}(H)$ be the set of all $Z \in L\left(C^{n}, H\right)$ so that $I-Z^{*} Z$ is positive definite. If $\operatorname{dim} H<\infty$ the $D_{n}(H)$ exhaust the Cartan domains of type I in the sense of Hua [3]. Furthermore, $D_{1}(H)=B$.

Let $Q$ be the quadratic form on $H \times C^{n}$ defined by $Q(z, w)=\|z\|^{2}$ $-\|w\|^{2}, z \in H, w \in \mathbf{C}^{n}$. Let $U(H, n)$ be the space of continuous linear maps of $H \times C^{n}$ to $H \times C^{n}, T$, so that $Q(T(z, w))=Q(z, w), Q\left(T^{*}(z, w)\right)$ $=Q(z, w)$. It is not hard to show that the elements of $U(H, n)$ are invertible. Hence $U(H, n)$ is a subgroup of the general linear group of $H \times C^{n}$. If $g \in U(H, n)$, and

$$
g=\left[\begin{array}{ll}
A & B \\
C^{*} & D
\end{array}\right]
$$

where $A: H \rightarrow H, B, C: C^{n} \rightarrow H, D: C^{n} \rightarrow C^{n}$, then for each $Z \in D_{n}(H)$, $C^{*} Z+D$ is invertible.

We may define

$$
g \cdot Z=(A Z+B) \circ\left(C^{*} Z+D\right)^{-1} .
$$

THEOREM 3.1. Let $f: D_{n}(H) \rightarrow D_{n}(H)$ be a holomorphic self-equivalence. Then there is a $g \in U(H, n)$ so that $f(z)=g \cdot Z$ for all $Z \in D_{n}(H)$.

Theorem 3.1 is proved using a generalized Schwarz lemma (a slight generalization of a result of Harris [2]) for bounded, convex, circled domains in Banach spaces and some elementary Hilbert space theory to reduce many of the difficulties to finite dimensional problems. The proof in [1] also gives a direct proof of this result in the case $\operatorname{dim} H<\infty$. 
THEOREM 3.2. $D_{n_{1}}(H) \times \cdots \times D_{n_{k}}(H)$ is holomorphically equivalent with $D_{m_{1}}(H) \times \cdots \times D_{m_{r}}(H)$ if and only if $r=k$ and there is a permutation $\sigma$ of $(1, \cdots, k)$ so that $m_{i}=n_{\sigma i}, i=1, \cdots, k$.

A similar result is true allowing the Hilbert spaces in the products to be different. It must only be kept in mind that $D_{n}\left(\boldsymbol{C}^{k}\right)=D_{k}\left(\boldsymbol{C}^{n}\right)$.

4. $D^{\infty}$. Let $H=l^{2}$ be the space of all square summable complex sequences. Let $D^{\infty}=\left\{\left\{z_{n}\right\} \in l^{2}\left|\sup _{n}\right| z_{n} \mid<1\right\}$.

LEMMA 4.1. $D^{\infty}$ is not holomorphically equivalent to a bounded domain in $H$.

The importance of this result is that it shows that Siegel domains of type II (even I! that is, tubes over open convex cones without straight lines) are not necessarily equivalent to bounded domains.

The details of the results announced in $\S 3,4$ will appear in [1].

\section{REFERENCES}

1. S. Greenfield and N. Wallach, Automorphism groups of bounded domains in Banach space (to appear).

2. L. Harris, Schwarz's lemma in normed linear space, Proc. Nat. Acad. Sci. U.S.A. 62 (1969), 1014-1017.

3. L. K. Hua, Harmonic analysis of functions of several complex variables in the classical domains, Science Press, Peking, 1958; English transl., Transl. Math. Monographs, vol. 6, Amer. Math. Soc., Providence, R. I., 1963. MR 23 \#A3277; MR 30 \#2162.

4. C. L. Siegel, Symplectic geometry, Amer. J. Math. 65 (1943), 1-86; Academic Press, New York, 1964. MR 4, 242; MR 29 \#1362.

Rutgers University, New Brunswick, New Jersey 08903 